\title{
Postsession Dreaming in Neurofeedback as an Indication of Nondeclarative Learning
}

\author{
Revital Yonah \\ BetterFly Neurofeedback, Tel-Aviv, Israel \\ Private practice, Jerusalem, Israel
}

\begin{abstract}
Clients may report increased dreaming following neurofeedback sessions. Increased dreaming may not be strictly a side effect of training but may rather be a result of the nondeclarative learning accomplished in training. Research has demonstrated the connection between dreaming and consolidation of memory in both animals and human subjects. Rapid eye movement (REM) deprivation studies have shown the importance of REM sleep to the retention of newly learned skills. Other studies have shown that learning may increase the proportion of REM sleep on subsequent nights. More specifically, REM dreaming may be related to the consolidation of procedural, nondeclarative memory, the type of learning that occurs also during neurofeedback training. When a client reports increased nocturnal dreaming following a neurofeedback session, this may serve as a valuable early indication that their brain is responding to this type of training.
\end{abstract}

Keywords: REM sleep; dreaming; neurofeedback training; nondeclarative learning; implicit learning; procedural learning and memory

Citation: Yonah, R. (2020). Post-session dreaming in neurofeedback as an indication of nondeclarative learning. NeuroRegulation, 7(1), 27. https://doi.org/10.15540/nr.7.1.2

*Address correspondence to: Revital Yonah, The Jerusalem Neurofeedback Clinic, P.O.B. 18144, Jerusalem, 9118101, Israel.

Email: RevitalYonah@gmail.com

Copyright: (C) 2020. Yonah. This is an Open Access article distributed under the terms of the Creative Commons Attribution License (CCBY).

\section{Background}

Neurofeedback is a form of neurotherapy in which the brain learns a new mode of operation. This learning is achieved as the brain gets feedback for its electrophysiological activity: positive feedback for the desired brainwave activity and negative feedback for the nondesired brainwave activity. Using this feedback, the brain learns to modify its pattern of electrophysiological activity. Most often, the feedback is given to clients in the form of sensory alterations during the session (i.e., visual and/or auditory changes to a video that the client is watching). In order to actually learn this new mode of operation and modify its electrophysiological activity in the desired direction, the brain has to understand the "language" of feedback - that is, it has to realize that there is actually a connection between its own brainwave activity and what is happening to the video being watched or the game being played on the
Edited by:

Rex L. Cannon, PhD, SPESA Research Institute, Knoxville, Tennessee, USA

Reviewed by:

Rex L. Cannon, PhD, SPESA Research Institute, Knoxville, Tennessee, USA

Randall Lyle, PhD, Mount Mercy University, Cedar Rapids, lowa, USA computer. It then has to learn that this connection is causal-that is, the sensory changes, which are perceived to be pleasant or unpleasant (positive or negative feedback, respectively), are contingent on the electrophysiological activity that it produces at any given moment during session. Finally, it has to devise a way to change its pattern of activity in accordance with this feedback.

Many clients have reported changes in cognition, mentation, and emotions following neurofeedback sessions; some of the changes are perceived as positive and desirable while others are less so. In the current paper we would like to discuss the oftenreported effect of increased dreaming following neurofeedback sessions and suggest that this may not be simply a side effect of training, but rather an indication that nondeclarative learning has taken place during the training session. 


\section{Increased Dreaming Following a Neurofeedback Session}

Some clients experience enhanced nocturnal dreaming following neurofeedback sessions (Gruzelier, 2009; Johnson, 2011; Larsen \& Verner, 2017; Leong et al., 2018a; Leong et al., 2018b). Reports of increased dreaming tend to be more common after a client's first few neurofeedback sessions, especially if the sessions are relatively long (30-40 minutes), although some clients may experience increased dreaming also through later stages of the training series and with shorter session durations. Dreams are reported to increase in quantity (more dreams than usual) and in quality (the dreams are reported to be enhanced: more vivid, more elaborate, and richer in detail than usual). Their contents may be experienced as emotionally positive or negative by the client, but the impression that they leave is strong enough for the client to at least remember having dreamed. Some clients report that while they normally experience and remember dreaming only in the early hours of the morning-just before waking up (which is in line with the fact that the late stages of a night sleep have a much higher proportion of REM sleep)-after the first neurofeedback sessions they experience dreaming "all through the night." This increased dreaming may be perceived as an adverse side effect by some, while others experience it as a positive phenomenon which they are excited to yield to and explore.

Protocol type may not be a determining factor when it comes to increased postsession dreaming. From our clinical experience, increased vivid dreaming may occur with different kinds of protocols. It has occurred when the sensors were placed frontally, centrally, or parietally, with both unipolar or bipolar electrode placements and with various protocols inhibiting or rewarding different frequency bands. A survey of existing literature reveals that increased dreaming occurred also with the alpha/theta protocol (Gruzelier, 2009; Johnson, 2011), with infra-slow neurofeedback (Leong et al., 2018a) and more.

It seems that rather than the type of protocol, the duration of each training session may be a more crucial factor. Long (30- to 40-minute sessions) may produce increased dreaming already after the first session, whereas shorter sessions (10 or 20 minutes) may produce increased dreaming only after four or five sessions (Leong et al., 2018a). Some clients report feeling more rested after such nights, while others may experience it as troubled sleep. Either way, it seems that increased dreaming following sessions may not be simply a side effect, but rather a positive indication that learning has been attained during the training. Increased postsession nocturnal dreaming may serve as an indication that the brain has done some procedural, nondeclarative learning during the session. In other words, increased vivid dreaming following a session may serve as an early indication that the brain is responding to this type of training-that it "understands the language" of feedback, so to speak.

Why would increased dreaming following a neurofeedback session be an indication of the brain responding to the neural feedback? We will explore this question next.

\section{Dreaming as an Indication of Learning}

Dreaming is a natural part of sleep in humans, and REM sleep occurs also through large parts of the animal kingdom (Ayala-Guerrero, Mexicano, \& Ramos, 2003; Dement, 1958; Peever \& Fuller, 2016). Some people claim to have never dreamed, but research has shown that unless a specific brain damage is present, all of us do, in fact, dream (Herlin, Leu-Semenescu, Chaumereuil, \& Arnulf, 2015). While dreaming is often associated with the REM stage of sleep (Aserinsky \& Kleitman, 1953; Dement \& Kleitman, 1957), it also occurs in non-REM sleep (Cavallero, Cicogna, Natale, Occhionero, \& Zito, 1992; Foulkes, 1962). According to different studies, the incidence of dream reports is between $70 \%$ and $90 \%$ when subjects are woken from REM sleep, but it drops dramatically when subjects are woken from non-rapid eye movement (NREM) sleep (Stoerig, 2007). While there is still some controversy among researchers as to the connection between REM sleep and dreaming, it seems to be the case that, although REM and dreaming are not the same, REM sleep is, indeed, accompanied by the subjective experience of dreaming: not only does the rate of reports of dreaming increases during REM sleep, but the reports of REM dreams tend to be longer, richer in detail, and more elaborate than those of NREM dreams (Takeuchi, Miyasita, Inugami, \& Yamamoto, 2001).

There are different competing theories concerning the function and role that dreaming serves, but among the ones that have attracted most attention are the theories postulating that dreaming is crucial for learning and memory consolidation (Poe, Walsh \& Bjorness, 2010; cf. Siegel, 2001). REM sleep deprivation studies have demonstrated the connection between learning and dreaming. In one 
early study, Pearlman (1969) showed that rats who were trained for an avoidance response and were subsequently REM sleep deprived did not demonstrate retention of that learning. Similarly, Fishbein (1971) found that depriving mice of REM sleep after a discrimination training in a maze prevented retention of that learning (Zhang et al., 2014). Detrimental effects of REM sleep deprivations on learning have been demonstrated in human subjects as well: Karni, Tanne, Rubenstein, Askenasy, and Sagi (1994) found that selective disruption of REM sleep resulted in no performance gain after learning a basic visual discrimination task (cf. Rasch, Pommer, Diekelmann, \& Born, 2009).

Another paradigm of research has been to measure the increases in REM sleep on subsequent nights following learning. In an animal study, Fishbein, Kastaniotis, and Chattman (1974) showed that mice who were trained in a shock-avoidance task displayed subsequent augmentations in slow wave sleep (SWS) and REM sleep, whereas their yoked controls showed augmentations only in SWS. In a study with human subjects, Maquet et al. (2000) showed that brain regions which were active during a serial reaction time task were also active during subsequent REM sleep, suggesting that REM sleep is involved in the reprocessing of memory traces. The same group (Peigneux et al., 2003) showed that the reactivation of cerebral regions during posttraining REM sleep is related to an implicit acquisition of the probabilistic rules that governed the sequence of stimuli during the serial reaction-time task, and that the extent to which the learning of probabilistic rules was attained is correlated with increases in regional cerebral blood flow during subsequent REM sleep.

While conflicting opinions exist among researchers as to the role of REM sleep in memory consolidation (see Rasch \& Born, 2013, for a review), it seems that there is ample evidence to suggest that REM sleep may be related to the consolidation of nondeclarative memory in particular. In one study, Mednick, Nakayama, and Stickgold (2003) had subjects learn a nondeclarative visual discrimination task. They found that those subjects who took a nap and had a bout of REM sleep after learning the task performed better in a subsequent test than those who slept only SWS, and their performance was even better than those who did not take a nap at all. This study demonstrates the importance of engaging in REM sleep to consolidate the learning of procedural, nondeclarative skills. Similarly, Tucker et al. (2006) found that a nap consisting of only SWS without bouts of REM sleep improved subjects' performance only on a declarative memory task but not on a nondeclarative task, which further strengthens the assumption that there is a dichotomy in memory processing during sleep between declarative and nondeclarative memory (i.e., the dual-process hypothesis). Furthermore, Plihal, and Born (1997) found that the first half of the night (which contains a larger proportion of SWS) is related to the processing of declarative memory, whereas the second half of the night (characterized by a larger proportion of REM sleep) is related to procedural, nondeclarative memory processing. Finally, REM sleep was found to improve performance in other types of learning as well, such as intensive language learning (De Koninck, Lorrain, Christ, Proulx, \& Coulombe, 1989), but these kinds of learning may have an implicit, nondeclarative memory component (Peigneux, Laureys, Delbeuck, \& Maquet, 2001).

\section{Neurofeedback as a Nondeclarative Learning-based Neurotherapy Method}

Neurofeedback is a learning-based neurotherapy method. It is considered to be a process that operates on the principles of operant conditioning (Hammond, 2011), in which the brain has to learn the connection between its electrophysiological activity and the sensory feedback it gets in return. After establishing the causality and directionality of this connection, the brain has to learn how to change its activity patterns to win more positive feedback and less negative feedback. This type of learning falls under the category of implicit, procedural, nondeclarative learning, and as such may be accompanied by more REM sleep periods on subsequent nights, which may be characterized by increased and enhanced vivid dreaming.

The first couple of neurofeedback sessions may be especially taxing for the brain in this regard, for the novelty involved: the brain first has to learn that there is a connection between what it does and the kind of experience it gets in return through the computer (a momentarily rewarding or a momentarily frustrating experience); it has to learn that the changes in sound and picture quality (or other forms of feedback) are directly related to its electrophysiological activity and that they serve, in fact, as feedback for its electrophysiological activity; and it has to learn how to change its activity in order to win more positive feedback and less negative feedback. Making these associations requires the brain to notice the subtle changes of its own brainwave activity, as well as the changes in the sensory experience during session. The brain has to try to fathom the direction in which this connection works-in other words, it has to 
realize that an increase or decrease in amplitude of certain brainwave frequencies brings about a negative (frustrating) feedback or a positive (rewarding) feedback. On top of it all, in these initial couple of sessions, the brain experiences its first attempt at trying to actually change its brainwave activity in response to this type of feedback. This entails a considerable amount of learning of the implicit, nondeclarative type; and, if the first session is long enough (30 to 40 minutes each), some clients may report substantially more dreaming and an enhanced vividness to their dreams already after the first session.

Our experience shows that most reports about increased dreaming following the first few neurofeedback sessions are done by adult clients rather than by young children. The reason may be that children's ability to be aware of and verbally describe such subjective experiences is less developed than that of the average adult, and this may make it harder for them to consciously access such experiences and relate them to others. Another possible explanation is that children spend a considerably higher proportion of their sleep cycles in REM sleep anyway, so the relative increases in REM sleep in their sleep due to neurofeedback training may be smaller.

\section{Discussion and Conclusions}

The field of sleep effects on learning and memory consolidation is a fascinating one, with a substantial increase in recent years in studies and theories attempting to explain the effects of the different stages of sleep on memory processing, facilitation, and consolidation. Although REM sleep has been implicated in the processing of other kinds of memory, especially in the processing of emotional memories (Groch, Wilhelm, Diekelmann \& Born, 2013; Nishida, Pearsall, Buckner, \& Walker, 2009; Wiesner et al., 2015), significant attention has been given to the study of REM sleep effects on implicit, nondeclarative learning.

Some researchers (Diekelmann, Wilhelm, \& Born, 2009; Marshall \& Born, 2007; Mednick et al., 2003; Smith, 1995) have shown support for the dualprocess hypothesis, by which declarative memory is processed mostly during SWS, whereas nondeclarative memory is processed mostly during REM sleep. Other researchers (Diekelmann \& Born, 2010; Ficca \& Salzarulo, 2004; Gais, Plihal, Wagner, \& Born, 2000; Giuditta, 2014) support the sequential processing hypothesis, by which both SWS and REM sleep are needed sequentially for the processing and consolidation of different types of memory, including procedural, nondeclarative memory.

Still, other suggestions have been made: it has been theorized that task complexity may be a determining factor, with more complex tasks being more sensitive to REM sleep deprivation (Walker, Brakefield, Morgan, Hobson, \& Stickgold, 2002). Interestingly, Ribeiro and Nicolelis (2004) postulate that the consolidation of implicit memories tends to be more complex, and therefore slower, than that of explicit memories, requiring a larger number of synaptic modifications. It could be that implicit learning of the type achieved in neurofeedback training may be such a complex, elaborate process and therefore may require REM sleep to be achieved.

Of special interest is the finding by Peigneux et al. (2003) cited above, that cerebral reactivation during posttraining REM sleep was related to the attainment of probabilistic rules that governed the sequences of stimuli in a serial reaction-time task. Implicitly extracting and learning probabilistic rules is something that the brain must perform during a neurofeedback session. That is, the client's brain must fathom the probability of getting positive feedback and negative feedback for changes in amplitude in the different frequency bands in order to modify its activity accordingly. REM sleep with its accompanying subjective experience of vivid dreaming may be involved in the processing of such higher-order information, embedded in this feedbackbased learning paradigm.

Without attempting to settle the discrepancy between the competing findings and theories concerning the role that different sleep stages play in memory consolidation, it seems that there is ample evidence obtained by various experimental methods in both humans and animals suggesting that REM sleep, which is characterized by the subjective experience of vivid, story-like dreaming, may be related to the processing and consolidation of nondeclarative learning and memory. Even when considering the conflict between the dual-process hypothesis and the sequential processing hypothesis, it seems that both of these theories assign importance to REM sleep when it comes to the processing of nondeclarative memory.

Our clinical experience (as well as that of other clinicians, as cited above) has shown that some clients report increased vivid dreaming following their first few neurofeedback sessions. Since 
neurofeedback is based on an implicit, nondeclarative type of learning, the ensuing increase in dreaming may serve as an indication that the client's brain is processing and consolidating the products of learning obtained during training.

Neurofeedback is an efficient neurotherapy method with high success rates; yet, as it is with any form of therapy, a certain percentage of clients (fortunately, not a big one) do not respond to it. Having some initial indication that a client is a responder may be an encouraging early sign for both clinician and client, which may boost the client's motivation to persevere in performing sessions twice or more weekly for the critical first few weeks of training. If our clients report increased and enhanced dreaming on the subsequent nights following their first neurofeedback session, we may assume that their brains "understand" the language of feedback and are responding to it. Now it is our job to fine-tune our protocol selection to make sure that they get optimal results.

It is important to note that not having any increase in dreaming or enhancement of dream vividness on subsequent nights following neurofeedback sessions does not necessarily mean that no learning has occurred or that the client's brain does not respond to the training. Dreaming following sessions is not a prerequisite for success in training. But when increased vivid dreaming does occur, then we have an initial indication that the brain "understands the language" of feedback and is responding to it. It would be a good practice to ask our clients after each session about their sleep quality and if they have noticed any changes in this regard.

Lastly, we believe that effort should be made to conduct controlled studies to investigate the effects of neurofeedback on REM sleep in sleep laboratories, as well as to find the relationship between the occurrence of increased REM sleep following sessions and the overall success in training. Such research may, in turn, shed further light upon the question of REM sleep effects on nondeclarative memory processing and consolidation.

\section{Author Disclosure}

The author has no grants, financial interests, or conflicts to disclose.

\section{References}

Aserinsky, E., \& Kleitman, N. (1953). Regularly occurring periods of eye motility, and concomitant phenomena, during sleep. Science, 118(3062), 273-274. https://doi.org/10.1126 /science.118.3062.273

Ayala-Guerrero, F., Mexicano, G., \& Ramos, J. I. (2003). Sleep characteristics in the turkey Meleagris gallopavo. Physiology \& Behavior, 78(3), 435-440. https://doi.org/10.1016/S00319384(03)00032-5

Cavallero, C., Cicogna, P., Natale, V., Occhionero, M., \& Zito, A. (1992). Slow wave sleep dreaming. Sleep, 15(6), 562-566. https://doi.org/10.1093/sleep/15.6.562

De Koninck, J., Lorrain, D., Christ, G., Proulx, G., \& Coulombe, D. (1989). Intensive language learning and increases in rapid eye movement sleep: Evidence of performance factor. International Journal of Psychophysiology, 8(1), 43-47. https://doi.org /10.1016/0167-8760(89)90018-4

Dement, W. C. (1958). The occurrence of low voltage, fast, electroencephalogram patterns during behavioral sleep in the cat. Electroencephalography and Clinical Neurophysiology, 10(2), 291-296. https://doi.org/10.1016 /0013-4694(58)90037-3

Dement, W. C., \& Kleitman, N. (1957). Cyclic variations in EEG during sleep and their relation to eye movements, body motility, and dreaming. Electroencephalography and Clinical Neurophysiology, 9(4), 673-690. https://doi.org/10.1016 10013-4694(57)90088-3

Diekelmann, S., \& Born, J. (2010). The memory function of sleep. Nature Reviews Neuroscience, 11(2), 114-126. https://doi.org /10.1038/nrn2762

Diekelmann, S., Wilhelm, I., \& Born, J. (2009). The whats and whens of sleep-dependent memory consolidation. Sleep Medicine Reviews, 13(5), 309-321. https://doi.org/10.1016 /j.smrv.2008.08.002

Ficca, G., \& Salzarulo, P. (2004). What in sleep is for memory. Sleep Medicine, 5(3), 225-230. https://doi.org/10.1016 /j.sleep.2004.01.018

Fishbein, W. (1971). Disruptive effects of rapid eye movement sleep deprivation on long-term memory. Physiology \& Behavior, 6(4), 279-282. $\quad$ https://doi.org/10.1016/00319384(71)90155-7

Fishbein, W., Kastaniotis, C., \& Chattman, D. (1974). Paradoxical sleep: Prolonged augmentation following learning. Brain Research, 79(1), 61-75. https://doi.org/10.1016/00068993(74)90566-6

Foulkes, W. D. (1962). Dream reports from different stages of sleep. The Journal of Abnormal and Social Psychology, 65(1), 14-25. https://doi.org/10.1037/h0040431

Gais, S., Plihal, W., Wagner, U., \& Born, J. (2000). Early sleep triggers memory for early visual discrimination skills. Nature Neuroscience, 3(12), 1335-1339. https://doi.org/10.1038 $/ 81881$

Giuditta, A. (2014). Sleep memory processing: The sequential hypothesis. Frontiers in Systems Neuroscience, 8, 219. https://doi.org/10.3389/fnsys.2014.00219

Groch, S., Wilhelm, I., Diekelmann, S., \& Born, J. (2013). The role of REM sleep in the processing of emotional memories: Evidence from behavior and event-related potentials. Neurobiology of Learning and Memory, 99, 1-9. https://doi.org/10.1016/j.nlm.2012.10.006

Gruzelier, J. (2009). A theory of alpha/theta neurofeedback, creative performance enhancement, long distance functional connectivity and psychological integration. Cognitive Processing, 10(1), 101-109. https://doi.org/10.1007/s10339008-0248-5

Hammond, D. C. (2011). What is neurofeedback: An update. Journal of Neurotherapy, 15(4), 305-336. https://doi.org $/ 10.1080 / 10874208.2011 .623090$ 
Herlin, B., Leu-Semenescu, S., Chaumereuil, C., \& Arnulf, I. (2015). Evidence that non-dreamers do dream: A REM sleep behaviour disorder model. Journal of Sleep Research, 24(6), 602-609. https://doi.org/10.1111/jsr.12323

Johnson, M. L. (2011). Relationship of alpha-theta amplitude crossover during neurofeedback to emergence of spontaneous imagery and biographical memory (Doctoral dissertation). University of North Texas, Denton, TX.

Karni, A., Tanne, D., Rubenstein, B. S., Askenasy, J. J., \& Sagi, D. (1994). Dependence on REM sleep of overnight improvement of a perceptual skill. Science, 265(5172), 679-682. https://doi.org/10.1126/science.8036518

Larsen, S., \& Verner, T. (2017). The transformational power of dreaming: Discovering the wishes of the soul. Rochester, VA: Inner Traditions Bear and Company.

Leong, S. L., Vanneste, S., Lim, J., Smith, M., Manning, P., \& De Ridder, D. (2018a). A randomised, double-blind, placebocontrolled parallel trial of closed-loop infraslow brain training in food addiction. Scientific Reports, 8(1), 11659. https://doi.org/10.1038/s41598-018-30181-7

Leong, S. L., Vanneste, S., Lim, J., Smith, M., Manning, P., De Ridder, D., \& Stoeckl-Drax, T. (2018b). Effects and side effects of infraslow network neurofeedback (ISF-NF): A randomised double blind placebo controlled trial. International Journal of Psychophysiology, 131(Suppl.), S151. https://doi.org/10.1016 /j.ijpsycho.2018.07.402

Maquet, P., Laureys, S., Peigneux, P., Fuchs, S., Petiau, C., Phillips, C., ... Cleeremans, A. (2000). Experience-dependent changes in cerebral activation during human REM sleep. Nature Neuroscience, 3(8), 831-836. https://doi.org/10.1038 177744

Marshall, L., \& Born, J. (2007). The contribution of sleep to hippocampus-dependent memory consolidation. Trends in Cognitive Sciences, 11(10), 442-450. https://doi.org /10.1016/j.tics.2007.09.001

Mednick, S., Nakayama, K., \& Stickgold, R. (2003). Sleepdependent learning: A nap is as good as a night. Nature Neuroscience, 6(7), 697-698. https://doi.org/10.1038/nn1078

Nishida, M., Pearsall, J., Buckner, R. L., \& Walker, M. P. (2009). REM sleep, prefrontal theta, and the consolidation of human emotional memory. Cerebral Cortex, 19(5), 1158-1166. https://doi.org/10.1093/cercor/bhn155

Pearlman, C. A. (1969). Effect of rapid eye movement (dreaming) sleep deprivation on retention of avoidance learning in rats. Report No. 563. U.S. Naval Submarine Medical Center.

Peever, J., \& Fuller, P. M. (2016). The biology of REM sleep. Retrieved from https://www.ncbi.nlm.nih.gov/pmc/articles /PMC5846126/

Peigneux, P., Laureys, S., Delbeuck, X., \& Maquet, P. (2001). Sleeping brain, learning brain. The role of sleep for memory systems. NeuroReport, 12(18), A111-A124. https://doi.org /10.1097/00001756-200112210-00001

Peigneux, P., Laureys, S., Fuchs, S., Destrebecqz, A., Collette, F., Delbeuck, X., ... Maquet, P. (2003). Learned material content and acquisition level modulate cerebral reactivation during posttraining rapid-eye-movements sleep. Neurolmage, 20(1), 125-134. https://doi.org/10.1016/S1053-8119(03)00278-7

Plihal, W., \& Born, J. (1997). Effects of early and late nocturnal sleep on declarative and procedural memory. Journal of
Cognitive Neuroscience, 9(4), 534-547. https://doi.org /10.1162/jocn.1997.9.4.534

Poe, G. R., Walsh, C. M., \& Bjorness, T. E. (2010). Cognitive neuroscience of sleep. Progress in Brain Research, 185, 119. https://doi.org/10.1016/B978-0-444-53702-7.00001-4

Rasch, B., \& Born, J. (2013). About sleep's role in memory. Physiological Reviews, 93(2), 681-766. https://doi.org /10.1152/physrev.00032.2012

Rasch, B., Pommer, J., Diekelmann, S., \& Born, J. (2009). Pharmacological REM sleep suppression paradoxically improves rather than impairs skill memory. Nature Neuroscience, 12(4), 396-397. https://doi.org/10.1038 /nn.2206

Ribeiro, S., \& Nicolelis, M. A. L. (2004). Reverberation, storage, and postsynaptic propagation of memories during sleep. Learning \& Memory, 11(6), 686-696. https://doi.org/10.1101 $/ \mathrm{m} .75604$

Siegel, J. M. (2001). The REM sleep-memory consolidation hypothesis. Science, 294(5544), 1058-1063. https://doi.org $/ 10.1126 /$ science. 1063049

Smith, C. (1995). Sleep states and memory processes. Behavioural Brain Research, 69(1-2), 137-145. https://doi.org/10.1016/0166-4328(95)00024-N

Stoerig, P. (2007). Hunting the ghost: Toward a neuroscience of consciousness. In P. D. Zelazo, M. Moscovitch, \& E. Thompson (Eds.), Cambridge handbook of consciousness (pp. 707-730). Cambridge, England: Cambridge University Press.

Takeuchi, T., Miyasita, A., Inugami, M., \& Yamamoto, Y. (2001). Intrinsic dreams are not produced without REM sleep mechanisms: Evidence through elicitation of sleep onset REM periods. Journal of Sleep Research, 10(1), 43-52. https://doi.org/10.1046/j.1365-2869.2001.00237.x

Tucker, M. A., Hirota, Y., Wamsley, E. J., Lau, H., Chaklader, A., \& Fishbein, W. (2006). A daytime nap containing solely non-REM sleep enhances declarative but not procedural memory. Neurobiology of Learning and Memory, 86(2), 241247. https://doi.org/10.1016/j.nlm.2006.03.005

Walker, M. P., Brakefield, T., Morgan, A., Hobson, J. A., \& Stickgold, R. (2002). Practice with sleep makes perfect: Sleepdependent motor skill learning. Neuron, 35(1), 205211. https://doi.org/10.1016/s0896-6273(02)00746-8

Wiesner, C. D., Pulst, J., Krause, F., Elsner, M., Baving, L., Pedersen, A., ... Göder, R. (2015). The effect of selective REM-sleep deprivation on the consolidation and affective evaluation of emotional memories. Neurobiology of Learning and Memory, 122, 131-141. https://doi.org/10.1016 /j.nlm.2015.02.008

Zhang, J., Zhu, Y., Zhan, G., Fenik, P., Panossian, L., Wang, M. M., ... Veasey, S. (2014). Extended wakefulness: Compromised metabolics in and degeneration of locus ceruleus neurons. The Journal of Neuroscience, 34(12), 44184431. https://doi.org/10.1523/JNEUROSCI.5025-12.2014

Received: January 9, 2020

Accepted: February 6, 2020

Published: March 25, 2020 